
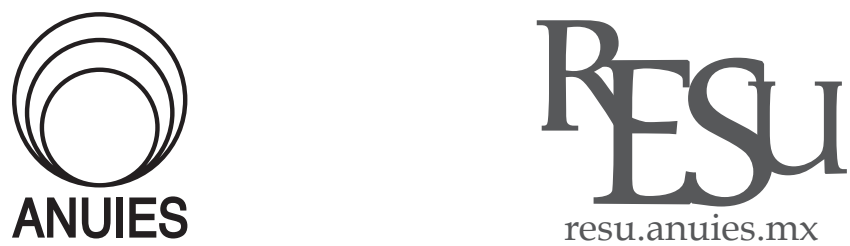

ARTíCULO

\title{
Los aprendices de brujos o los primeros acercamientos hacia la investigación. Un estudio sobre el programa del Verano de la Investigación Científica*
}

Sorcerers' apprentices or the first approaches to research. A study of the Scientific Research Summer program

\author{
Rocío Rosas Escamilla** y Alma Maldonado Maldonado*** \\ * Las autoras agradecen al programa Verano de la Investigación Científica por las facilidades proporcionadas \\ para el uso de sus bases de datos. \\ ** Programa transdisciplinario en Desarrollo Científico y Tecnológico para la Sociedad del Centro de \\ Investigación y de Estudios Avanzados del Instituto Politécnico Nacional. Correo electrónico: rrosas@cinvestav.mx \\ *** Departamento de Investigaciones Educativas del Centro de Investigación y de Estudios Avanzados del \\ Instituto Politécnico Nacional. Correo electrónico: almaldo2@gmail.com
}

Recibido el 02 de marzo del 2017; aceptado el 06 de marzo del 2018.

\section{Resumen}

Este artículo indaga en la experiencia que significa para un alumno participar en una estancia corta de investigación durante la licenciatura y, en general, aborda el tema de cómo se construye en los estudiantes el interés por la investigación como actividad profesional. Se analizan los datos del programa Verano de la Investigación Científica (VIC) y son contrastados con el directorio 2014 del Sistema Nacional de Investigadores (SNI). Se obtuvo un primer acercamiento sobre el destino de algunos ex participantes y el posible impacto de dicho programa en el desarrollo de las vocaciones relacionadas con el trabajo científico. Además, se incluyen los resultados de un componente cualitativo de la investigación cuyo propósito fue recuperar la experiencia y las reflexiones de algunos de estos sujetos, específicamente un grupo de investigadores del SNI que participaron en el programa del vic en el periodo de 1991 a 1995.

Palabras clave: Formación de investigadores; Investigación; Verano de la investigación científica; Desarrollo científico; Políticas científicas

0185-2760/@ 2016 Asociación Nacional de Universidades e Instituciones de Educación Superior A.C. Este es un artículo Open Access bajo la licencia CC BY-NC-ND (http://creativecommons.org/licenses/by-nc-nd/4.0/). 


\begin{abstract}
This paper discusses the relevance of participating in a short-term research mobility program conducted at Mexican higher education institutions. The "Verano de la Investigación Científica" (VIC) [Scientific Research Summer] program seeks to promote scientific activities (and eventually research vocations) among college students. This paper analyzes the degree to which the program serves as a feeder for the National System of Researchers (SNI), a pay-for-performance, peer-review system run by the Mexican government, by comparing the directories of both programs. It also presents data from a qualitative study in order to recover the experiences and voices of some current SNI members who participated in the Sumer Program from 1991 to 1995.
\end{abstract}

Keywords: Researchers' training; Research; Short-term mobility; Scientific development; Public policies on science

\title{
Introducción
}

$\mathrm{E}$ n "El aprendiz de brujo", J. W. Goethe cuenta la historia de un hechicero que se dedicaba al estudio de las fórmulas mágicas y que no permitía más visita que la de Daniel, su aprendiz. En una ocasión, el hechicero salió por varios días y le encomendó a Daniel que en su ausencia no entrara en su estudio y que limpiara algunas habitaciones. Después de algunos días de cumplir con el encargo del hechicero, Daniel desobedece y entra en el estudio e intenta hacer magia para que la escoba y el balde de agua limpien solos el lugar, pero su poca sapiencia provoca un desastre que sólo pudo controlarlo el hechicero, el cual aparece cuando el lugar está inundado y el aprendiz casi ahogándose. Este cuento recrea la relación entre un aprendiz y su maestro y la - a vecesnecesaria aceptación del aprendiz al sistema de normas que es comunicado por el maestro para que aquél pueda llegar a ser un hechicero también algún día.

La carrera académica es una profesión que mantiene algunas peculiaridades y diferencias respecto a otras profesiones. Como se ha escrito ampliamente, el trabajo de investigación requiere de una formación especializada que, en la actualidad, se obtiene cursando un doctorado. Parry (2007: 11) apunta que "en buena medida el doctorado es una prueba de madurez y de competencia intelectual y cultural", además "denota el dominio de una disciplina y la confianza y la agilidad en la producción y la difusión del conocimiento en un campo particular" (Parry, 2007: 3). Al mismo tiempo, en la literatura se ha discutido hasta qué punto la labor del investigador debe ser 
considerada como un oficio que se aprende más allá del trabajo de las aulas, dado que se trata de una actividad que se aprende en la práctica (igual que la docencia).

El presente artículo indaga en la experiencia del programa "Verano de la Investigación Científica", que es apoyado por la Academia Mexicana de las Ciencias y que ha impulsado a jóvenes interesados en la investigación para que prosigan con una carrera académica en el futuro. El programa también promueve la movilidad académica, pues busca que los muchachos tengan una experiencia de investigación en una entidad diferente a la que estudian, y explora cómo la movilidad geográfica brinda elementos de formación a los alumnos. Más adelante se advertirá que si bien diversos elementos impulsaron a los jóvenes en sus distintas áreas disciplinarias hacia el ejercicio profesional de la investigación, en principio, la interacción aprendiz-maestro resultó fundamental para conocer el significado de esta labor.

\section{La formación de investigadores y la ciencia en México}

La formación para la investigación busca facilitar — de manera sistematizada (no necesariamente ocurre en el sistema escolarizado de educación, aunque es la vía más usual) - el acceso a un conjunto de conocimientos, tanto de hábitos y de actitudes como el desarrollo de habilidades y la internalización de ciertos valores, medios requeridos para realizar la actividad de la investigación (Moreno, Sánchez, Arredondo, Pérez y Klingler, 2003; Guerrero, 2007). Adicionalmente hay otros elementos que promueven tal formación, por ejemplo, el contexto, el capital social y cultural de los individuos, las redes, "las lecturas, las circunstancias, los accidentes de vida", entre otros (Ferry, 1991: 55, citado en Moreno et al., 2003). En este proceso es posible identificar dos aspectos clave: 1) la intencionalidad, referida a la enseñanza y la apropiación de un habitus, es decir, el camino hacia el ejercicio de una profesión en cuyo tránsito se refleja la influencia de los formadores en los aprendices; y 2) la internalización del habitus como producto de la continua e intensa interacción entre expertos y aprendices.

La figura del académico ha sido muy importante para atraer a los estudiantes hacia la investigación y ha influido para que éstos se dediquen a ella (Stekolschik, Gallardo y Draghi, 2007, González, 2013). Ramírez (1998) se refiere al caso de los alumnos en la Facultad de Química de la UNAM y señala que si bien la formación del tipo aprendiz de "héroe" (hechicero) no forma parte del curriculum, sí resulta un factor importante para generar la curiosidad científica en los estudiantes, pues el respaldo de un investigador 
se vuelve un punto de partida para motivarlos a pensar, a mirar más allá, a inquirir, a crear, a concebir miles de cosas y, a la postre, puede convertirse en el mentor que los guíe en la toma de decisiones sobre su carrera académica. Otro tema que no puede ignorarse estriba en la dificultad de atraer jóvenes hacia la ciencia en las escuelas con menos recursos económicos, las cuales tienen menos vinculación con los centros de investigación, investigadores y universidades donde se genera el conocimiento (Zelzman, Francisco y Daraio, 2009). El tema de la inequidad y la formación de investigadores debería ser analizado con mayor detenimiento en un contexto como el mexicano, donde el capital cultural de los individuos aparece relacionado - aunque no siempre - con su nivel socioeconómico. No es requisito para dedicarse a la ciencia ser un individuo opulento, pero muchos de quienes terminan dedicándose a ella tuvieron las posibilidades económicas, la motivación y la orientación adecuadas para hacerlo. En este sentido, un programa como el Verano de la Investigación Científica puede erigirse en un espacio interesante para suplir algunas de esas carencias y darle la oportunidad a jóvenes de escasos recursos con inquietudes académicas para encauzar una decisión vocacional que de otra manera no podrían ejecutar.

De la revisión de la literatura se desprenden algunos temas relevantes, por ejemplo, Landesmann (2004) determinó tres generaciones de académicos en el departamento de Bioquímica de la Facultad de Medicina de la unAm: los "fundadores", los "herederos", y los "profesores de facultad". En la formación y la socialización de la generación de pupilos o "herederos", no sólo fueron relevantes los vínculos con los tutores o "fundadores", sino también con los pares. Los "herederos" valoraron haber contado con un espacio físico para trabajar $y$, sobre todo, con un ambiente de trabajo en el que tuvieron la oportunidad de conocer a un grupo de personas, como ellos, interesadas en la ciencia. Por su parte, Rosas (2006) estudió a un grupo de expertos pertenecientes al subsistema de investigación científica de la UNAM, con más de 30 años de trayectoria, y la mayoría de ellos señaló que su inserción en la vida académica fue gracias a la influencia que recibieron de otros académicos a quienes conocieron y trataron durante sus estudios de licenciatura y de posgrado; otros hallazgos apuntaron que más de la mitad de ellos seguía en contacto con dichos académicos; hubo también una muy baja presencia de mujeres académicas como modelo a seguir.

Los espacios materiales con una cultura de generación de conocimientos, la voluntad política para el fomento a la investigación, la constante interacción maestros-estudiantes, la articulación de los vínculos y la presencia de un tutor con trayectoria académica son algunos componentes que influyen para que un estudiante - desde su formación profesional universitaria - conozca 
y defina los términos "investigación" e "investigador" (Montiel, 2012); dentro de todos estos aspectos, la figura del tutor representa ese modelo "ideal" de lo que se quiere ser (Ramírez, 1998).

Menin (2000) concibe como "artesanal" el tipo de aprendizaje en el que se sigue y, a veces, se imita al maestro (¿al brujo?), ejecutando "paso a paso y acabadamente" la tarea de investigación que se le asigna al aprendiz. Según este modelo, usado entre los artesanos desde la Edad Media, así como entre los artistas, hechiceros, magos (¿alquimistas?), el arte y el oficio descansan en la práctica intensa y constante de la tarea a realizar. En dicho modelo, el conocimiento se trasmite socializándolo, lo cual ha contribuido históricamente en la formación de investigadores. De acuerdo con Candela (1999: 31), se trata de una forma de aprendizaje caracterizada por la "observación e imitación en el que un 'aprendiz' aprende colaborando en el trabajo especializado con el 'maestro' o el experto."

Si bien, el papel que puede ejercer el tutor - mentor, maestro - involucra una parte crucial en el proceso de formación de la investigación, otra parte importante radica en las condiciones materiales, los apoyos y la infraestructura con la que cuentan estos aprendices y sus maestros en el proceso. En este terreno se enmarca el análisis del programa Verano de la Investigación Científica, creado por la Academia Mexicana de Ciencias, A.C., en el año de 1991 en México. Este programa promueve y apoya a estudiantes de licenciatura para que realicen estancias cortas de investigación con el propósito de que conozcan la actividad en su contexto real; asimismo, permanece abierto a los estudiantes de cualquier institución de educación superior y carrera.

En el caso mexicano, las condiciones para la formación de investigadores son muy complicadas; de acuerdo con De la Fuente (2010), existen tres características estructurales de la ciencia en México que contribuyen a ello: 1) baja inversión en ciencia y tecnología, 2) poca participación de la inversión privada, y 3) un sistema universitario desequilibrado y dominado por las ciencias sociales.

Sobre el primer punto, según Maldonado (2017), el análisis de ciertos indicadores sobre ciencia y tecnología se convierte en una forma de entender cómo es que los países se dividen en centrales, periféricos o marginales en la producción del conocimiento; entre tales indicadores aparece, por ejemplo, la inversión en ciencia y tecnología con respecto al Producto Interno Bruto (PIB) de cada país. La autora presenta el caso de los Estados Unidos, puesto que ningún país alcanza sus niveles de inversión en ciencia y desarrollo: en 2013 destinó casi 433 mil millones de dólares, aun cuando su proporción con respecto al PIB fue de $2.81 \%$, porcentaje que quedó por debajo de Israel y de Corea que para el mismo año reportaron 4.21 y $4.15 \%$, respectivamente. 
Esta proporción del PIB en el gasto en ciencia y tecnología ofrece una idea de la importancia que cada país otorga a la ciencia y el desarrollo. En México, el gasto federal en ciencia y tecnología nunca ha alcanzado, desde 1990, la representación del 0.5\% como proporción del PIB (Conacyt, 2002: 14, Conacyt, 2009: 249, Conacyt, 2012: 18, Conacyt, 2015: 197, Conacyt, 2017: 24), mientras que para el caso del Gasto en Investigación y Desarrollo Experimental (GIDE), el 0.5\% se alcanzó apenas en el año 2009 (Conacyt, 2009: 384, Conacyt, 2015: 189, Conacyt, 2017: 217). A nivel internacional, este bajo porcentaje coloca a México entre los últimos lugares de los países miembros de la OCDE (Conacyt, 2014: 294), junto a otros como Argentina, Chile, Grecia, Turquía y Sudáfrica, que reportan proporciones menores a un $1 \%$. También el número de investigadores por cada mil integrantes de la Población Económicamente Activa (PEA), en nuestro país ronda la cifra de uno, con algunas muy leves variaciones (Conacyt, 2009: 388, Conacyt, 2014: 299); esta proporción nos sitúa de nuevo junto a países como Chile o Sudáfrica, los cuales también rondan o superan ligeramente la razón de un investigador, y al mismo tiempo ejemplifica la franca desventaja del país con respecto a Corea del Sur, Finlandia, Japón, Noruega, Portugal y Suecia, los cuales superan los 10 investigadores por cada mil de la PEA, y se trata de los países (con excepción de Portugal con un $1.5 \%$ ) con una mayor proporción de GIDE con respecto al PIB. Por lo que se refiere al impacto de artículos publicados, tampoco ocupamos una posición favorable a escala internacional (Maldonado, 2017): la ocDE estableció que un país es reconocido como un nodo de intercambio científico mundial si reportaba más de 200000 citas entre 1996 y 2013, mientras que los países que lograran más de 100000 serían considerados puntos de referencia del flujo de citas. En el caso de América Latina, sólo Brasil se percibió como un punto de flujo con los Estados Unidos, pero Argentina, Chile y México no alcanzaron las 100000 citas requeridas, por lo que aparecieron como puntos aislados.

Respecto al segundo punto destaca la poca participación de la inversión privada. El gasto en investigación y desarrollo experimental viene financiado en principio por la aportación del gobierno y luego por el sector productivo; le siguen en menor medida los sectores educación superior, privado no lucrativo y los fondos del exterior. El sector productivo reportaba una participación de apenas 14\% en 1993 (Conacyt, 2002: 18) y para 2011 ascendió a 37\%; su máximo nivel fue en los años 2006 y 2007, en los cuales alcanzó 45\% (Conacyt, 2012: 24). El personal dedicado a investigación y desarrollo experimental (IDE) emerge inserto laboralmente en el sector productivo, en el gobierno, en el sector educación superior y en el privado no lucrativo. En 1993 sólo 7\% del personal dedicado a IDE trabajaba en el sector productivo 
(Conacyt, 2002: 36), y este porcentaje se incrementó para el año 2011 a 38\%, alcanzando su máximo entre los años 2005 y 2007, en los cuales llegó a reportar 51\% de absorción (Conacyt, 2012: 42).

En cuanto al tercer punto, México cuenta con un sistema universitario dominado en términos de matrícula por las ciencias sociales. Las razones de esta distribución son muchas, pero se relacionan con la complejidad de desarrollar programas relacionados con ciencia (a nivel nacional e institucional) y en los retos que una carrera científica puede representar a nivel individual (laboral, profesional y personalmente). En la licenciatura, las áreas disciplinarias que concentran la matrícula son ciencias sociales, administración y derecho con $42 \%$, e ingeniería, manufactura y construcción con un $27 \%$; siguen, en menor medida, las ciencias de la salud con $10 \%$ y educación con $8 \%$, y al final se encuentran las ciencias naturales, exactas y de la computación, artes y humanidades, agronomía y veterinaria con 6\%,4\% y 2\% respectivamente (ANUIES, 2014). Asimismo, además de la distribución entre las áreas del conocimiento, también existen desequilibrios geográficos e institucionales importantes.

De la Fuente (2010: 3) apunta que la formación para la investigación en México se lleva a cabo en "instituciones de educación superior, centros Conacyt, centros e institutos del gobierno y del sector paraestatal, y en algunos centros y laboratorios del sector productivo", y considera que, si bien la formación para la investigación en las principales universidades del país - la Universidad Nacional Autónoma de México (UnAM), el Instituto Politécnico Nacional (IPN) y la Universidad Autónoma Metropolitana (UAM) - puede llegar a ser más exigente y de mayor rigor, la posibilidad de acceder al mercado de trabajo académico se incrementa considerablemente si los estudios de posgrado, necesarios para formarse como investigador, se realizaron en el extranjero.

$\mathrm{Al}$ parecer las becas para estudiar en el extranjero todavía representan un bien escaso, ya que en poco más de veinte años (desde 1990 hasta el 2012) las becas nacionales otorgadas por el Conacyt superan en poco más de cinco veces a las becas para estudiar en el extranjero: 377849 becas nacionales contra 68 869 becas al extranjero. También se observa una clase de centralización del posgrado y la investigación en el país: en 2014 casi $24 \%$ de la matrícula nacional de maestría y de doctorado se concentró en Ciudad de México, seguida en menor medida por el Estado de México y Puebla con 9.8 y 7.8\% respectivamente. Por lo que se refiere a las instituciones, el $7 \%$ de la matrícula nacional de maestría y de doctorado se ubicó en la UNAM, mientras que el IPN y la UAM representaron el 2 y el 1\% respectivamente. No es de extrañar entonces que 35\% de los 
miembros del directorio 2014 del Sistema Nacional de Investigadores (SNI) se ubiquen en Ciudad de México y 18\% del total pertenezcan a la UNAM.

\section{El programa del Verano de la Investigación Científica}

Grediaga (2012) señala que la formación de un investigador inicia con el desarrollo de los estudios superiores; éstos representan la inversión de tiempo y de recursos que el individuo decide realizar para conocer el estado del conocimiento en la línea de investigación de su interés, adquirir las herramientas y pautas de interacción con otros miembros de la comunidad, y así ser considerado como aspirante para formar parte de la disciplina en la que desea insertarse. Los estudios superiores representan los primeros mecanismos de socialización en el ámbito de la investigación.

Por lo que se refiere al reclutamiento de nuevos investigadores, García Salord (1999) resalta que, durante décadas, los académicos no han podido definir una estrategia de reproducción como grupo ocupacional que considere su naturaleza heterogénea. Sin embargo, la tradición de atraer y de formar jóvenes para la ciencia mediante el contacto con un experto o maestro (como aprendiz de investigador) continúa hasta nuestros días.

El Verano de la Investigación Científica es un programa de carácter nacional cuya dinámica coincide con la tradición señalada por García Salord (1999), la cual ha consistido en atraer a estudiantes de licenciatura hacia la investigación científica en cualquiera de sus áreas mediante la movilidad académica. El programa proporciona apoyo económico y logístico para que cierto número de estudiantes seleccionados realicen una estancia de dos meses (julio y agosto), bajo la guía de un académico activo en un centro de investigación del país. Dicho programa comenzó en 1991 con 99 participantes y a partir de ese año la cifra ha crecido gradualmente, sobresaliendo una presencia superior de las mujeres con respecto a sus compañeros varones, tema que debería estudiarse más a fondo, pues puede indicar la progresiva feminización de la matrícula de educación superior, o bien los cambios culturales en la sociedad mexicana producidos, por ejemplo, por los esfuerzos gubernamentales para atraer a las niñas y adolescentes a la ciencia, y en general, por las campañas que promueven el empoderamiento de las mujeres; además también debe influir el hecho de que, como se observa en la Tabla 1, la mayoría de los participantes del verano se ubican principalmente en las 
áreas de ciencias sociales y humanidades, y de ciencias biológicas, biomédicas y químicas, donde hay una mayor presencia femenina. La participación por área parece ser un reflejo de la matrícula nacional, aun cuando no se trata de una muestra representativa: las áreas de sociales y de humanidades son las que tienen el mayor porcentaje (33\%), pero los otros dos tercios se dividen entre las siguientes áreas: físico matemáticas, biológicas, biomédicas y químicas, e ingeniería y tecnología.

Tabla 1

Verano de la Investigación Científica, participantes 1991-2012 por sexo y área disciplinaria

\begin{tabular}{lccccc}
\hline & Mujeres & Hombres & Total & $\%$ de mujeres & $\%$ por disciplina \\
\hline Físico matemáticas & 698 & 1116 & 1814 & $38 \%$ & $11 \%$ \\
\hline Biológicas, biomédicas y químicas & 2649 & 1617 & 4266 & $62 \%$ & $26 \%$ \\
\hline Sociales y humanidades & 3874 & 1590 & 5464 & $71 \%$ & $33 \%$ \\
\hline Ingeniería y tecnología & 2140 & 2673 & 4813 & $44 \%$ & $29 \%$ \\
\hline Total & 9361 & 6996 & 16357 & $57 \%$ & $100 \%$ \\
\hline
\end{tabular}

Fuente: elaboración propia a partir de la base de datos del Verano de la Investigación Científica.

Los motivos por los que un estado envió más estudiantes que otro pueden ser muy variados: tamaño y número de Instituciones de Educación Superior, labor de difusión de cada coordinación estatal, respuesta de los estudiantes, etc. Entre 1991 y 2012, el estado de Sinaloa fue el que envió más estudiantes, seguido de Tabasco, 17\% y 10\% de los participantes respectivamente, mientras que la Ciudad de México y Veracruz contribuyeron con 9\% y 8\%. Por otro lado, los cuatro estados con menor representación fueron Morelos, Campeche, San Luis Potosí y Baja California Sur con porcentajes menores al 1\%.

Al ser un programa de movilidad académica, los estudiantes no tienen ninguna restricción en el momento de elegir al investigador y la institución en la que desean realizar su estancia; las únicas dos condiciones radican en que se lleve a cabo dentro de la República Mexicana y en una institución diferente a la suya. Los estudiantes han realizado sus estancias principalmente en la Ciudad de México, lo cual puede explicarse por la concentración de la investigación nacional en instituciones como la UNAM, el IPN, la UAM y el Centro de Investigación y de Estudios Avanzados del IPN (Cinvestav), además de los institutos nacionales de salud. 
Figura 1

Participantes del Verano de la Investigación Científica por estado de procedencia, 1991-2012

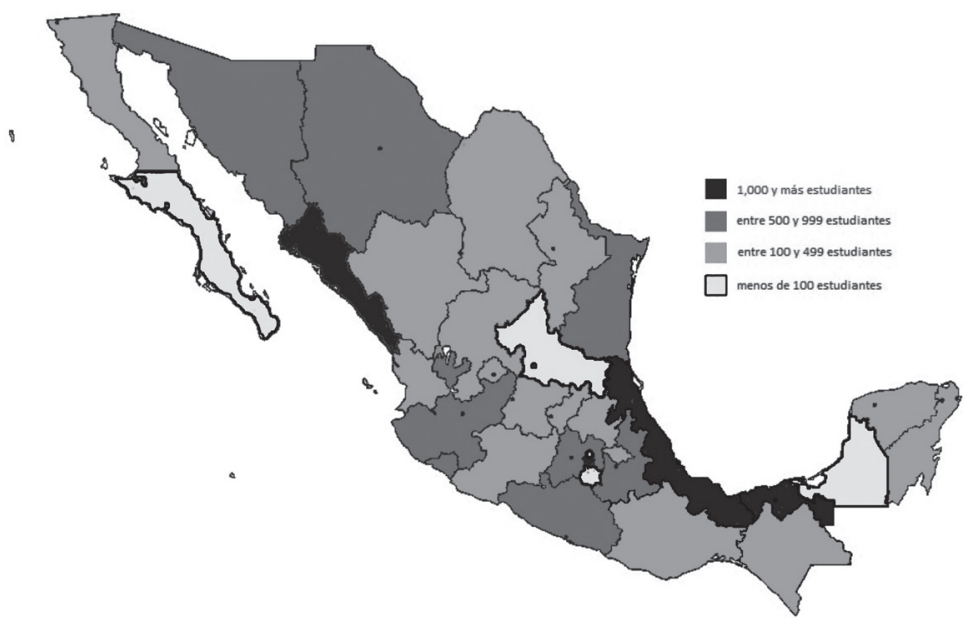

Fuente: elaboración propia a partir de la base de datos del Verano de la Investigación Científica.

Figura 2

Participantes del Verano de la Investigación Científica por estado de la estancia, 1991-2012

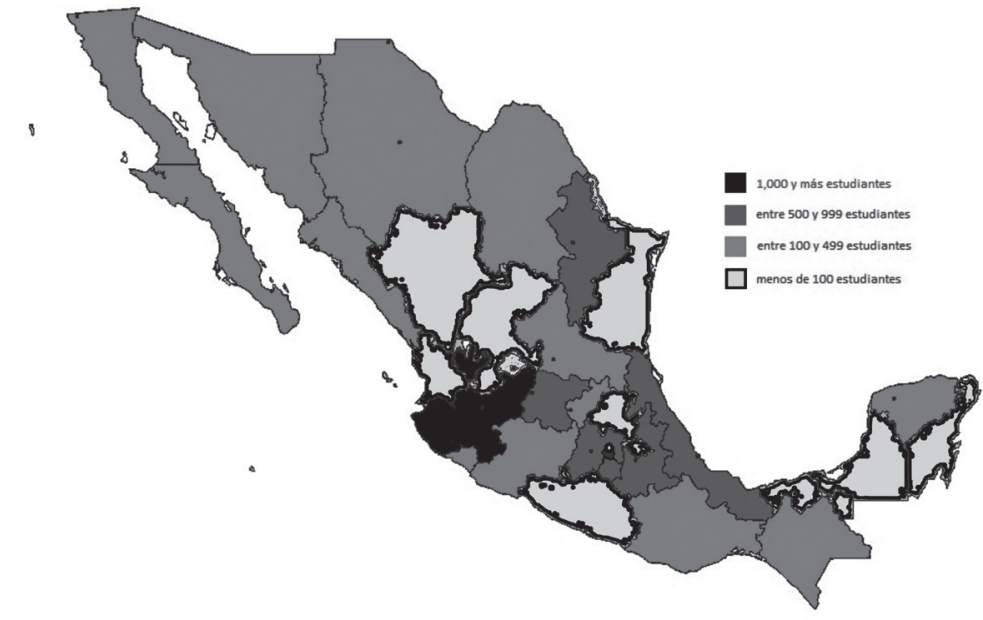

Fuente: elaboración propia a partir de la base de datos del Verano de la Investigación Científica.

Dicha tendencia ha ido cambiando en la medida que el programa es cada vez más conocido; un número cada vez mayor de instituciones van participando y también se van creando más instituciones en los estados. Con todo, la Ciudad de México representa la tercera parte del destino elegido por los estudiantes (Gráfica 1). 


\section{Gráfica 1}

Verano de la Investigación Científica, participantes que realizaron la estancia en Ciudad de México comparado con los demás estados de la República

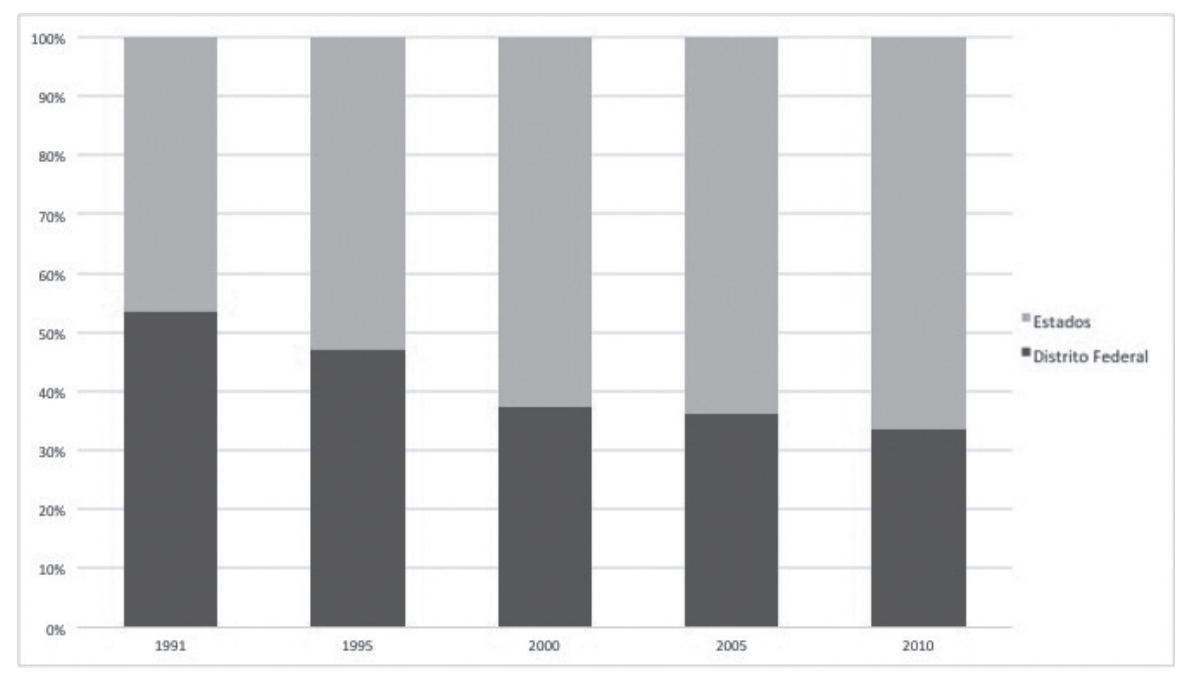

Fuente: elaboración propia a partir de la base de datos del Verano de la Investigación Científica.

El programa inspiró la creación de otros programas de alcance regional: en 1996 comenzó el Verano de la Investigación Científica y Tecnológica del Pacífico o Verano "Delfín", coordinado en el estado de Nayarit; hoy día puede contarse con el verano de la ciencia de la región centro (coordinado en Aguascalientes y creado en 1998), el programa de verano de estancias científicas y tecnológicas (coordinado en Chiapas y del que se desconoce el año de su creación), veranos por la innovación en la empresa (coordinado en Ciudad de México y del que se desconoce el año de su creación), el verano de la investigación científica de la Universidad de Guanajuato (creado en 1994); también existe el programa de estancias de investigación, desarrollo tecnológico e innovación del estado de Hidalgo (se desconoce el año de su creación); hay además las estancias estatales de investigación para estudiantes de nivel licenciatura (coordinado en Michoacán y creado en 2009), jóvenes talentos (coordinado en Quintana Roo y creado en 2011), el verano de la ciencia en la UASLP (la modalidad nacional creada en 2009) y el verano de la investigación científica de la península de Yucatán "Jaguar" (se desconoce el año de su creación).

Durante los primeros años del Verano de la Investigación Científica se llevó a cabo un congreso al final de la estancia; se trataba de un acto que congregaba a todos los participantes y algunos de sus investigadores, en el que los estudiantes presentaban los resultados de su investigación y a la vez tenían la oportunidad de interactuar y de socializar con otros estudiantes con 
intereses similares en la ciencia y la investigación. Este evento dejó de realizarse para destinar los recursos al otorgamiento de más becas, pero el caso concreto del Verano "Delfín" aún continúa llevando a cabo dicho congreso, lo que resulta muy atractivo para los alumnos. De hecho, hoy día, el Verano Delfín consta de tres subprogramas: Movilidad estudiantil (el Verano de la Investigación Científica y Tecnológica del Pacífico), Divulgación científica y tecnológica (mediante el cual se promueven tópicos de investigación y de la oferta educativa de posgrados) y Movilidad de profesores e investigadores (para el desarrollo de actividades de divulgación científica y tecnológica y de promoción del posgrado e investigación).

La información disponible, de los últimos ocho años del programa, permite mostrar, efectivamente, un incremento en el monto de los recursos destinados para becas, beneficiando a un número cada vez mayor de participantes, que tal vez responde a un cambio de estrategia. No obstante, si bien ha aumentado el apoyo económico, todavía son muchos alumnos los que, aun con una evaluación favorable, no tienen acceso a una beca. En ese sentido, sobresale el creciente apoyo económico que ofrecen las universidades a sus estudiantes, para que estén en condiciones de realizar su estancia, incluso si no acceden a una beca de la AMC.

Gráfica 2

Verano de la Investigación Científica, participantes por

fuente de fondos y monto anual de las becas

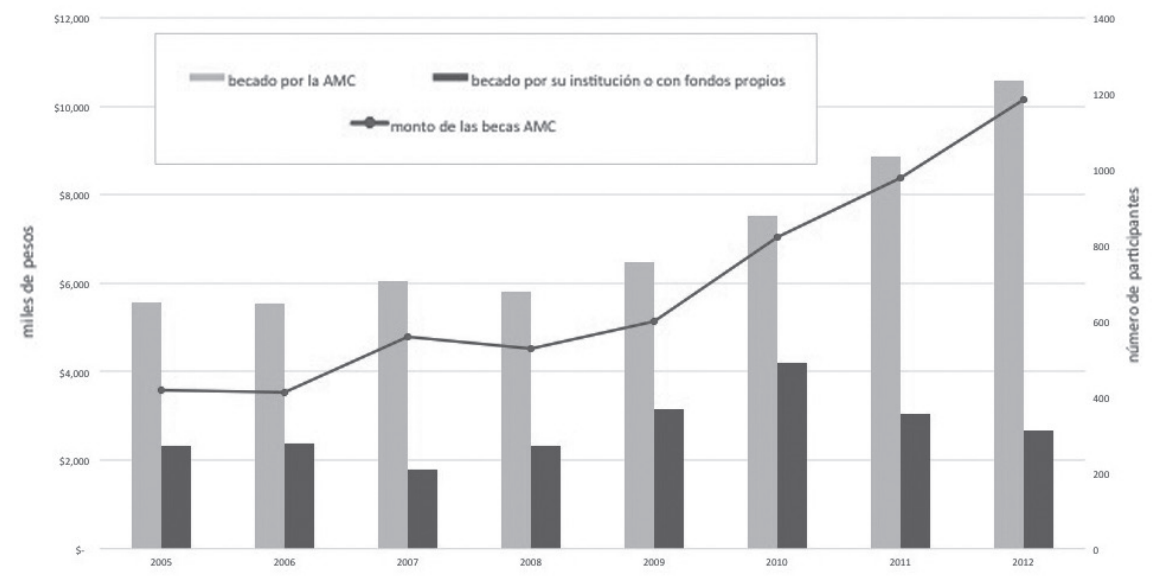

Fuente: elaboración propia a partir de la base de datos del Verano de la Investigación Científica.

Algunas universidades participantes han publicado artículos que confirman que la realización de la estancia en el Verano ha promovido en los estudiantes una real atracción hacia la actividad científica (Urrea, 2013; Sáenz, Her- 
nández y Delgado, 2013; Magaña, Aguilar, Pérez, Quijano y Argüelles, 2014), dado que cambian sus expectativas profesionales hacia la investigación y los estudios de posgrado (Urrea, 2013), e inclusive señalan la importancia que tuvo el asesoramiento del tutor para confirmar dicho interés; tales publicaciones subrayan también la necesidad de realizar análisis posteriores por medio de los cuales sea posible conocer si la realización de dichas estancias tuvieron un impacto en el interés científico de los escolares (Magaña et al., 2014), sin embargo, la apuesta a futuro está dirigida hacia la formación de más y mejores investigadores, ya que los propios participantes recomendarían a más estudiantes para que participen en el programa (Sáenz et al., 2013).

La información de la encuesta de salida que se ha aplicado en los últimos años del programa, desde el año 2002, indica que la estancia de investigación del vic generó en los estudiantes un genuino interés en la investigación, por la naturaleza de las actividades que realizaron, el apoyo y la asesoría recibidos por parte del investigador que los atendió y el acceso a instalaciones y materiales, expresando en la mayoría de los casos la intención de estudiar algún posgrado una vez concluida su carrera (Academia Mexicana de Ciencias, 2012). Posteriormente, el 12 de agosto de 2015 se celebró el xxv aniversario del Verano de la Investigación Científica en las instalaciones de la Academia Mexicana de Ciencias; el director del programa señaló que se aplicó una encuesta entre los participantes desde el año 2000 y hasta el 2015, de tal suerte que entre los resultados importantes se reportó que 67\% realizó o realiza estudios de posgrado, $72 \%$ evaluó al programa como excelente y $65 \%$ afirmó que fue decisivo para definir su futuro laboral en su disciplina (no se señaló el número de participantes ni de aquellos que respondieron). Sin embargo, poco se conoce de la situación laboral actual de los participantes y de las circunstancias que la rodean; tampoco se conoce hasta qué punto una experiencia como el verano influyó en la decisión de estos individuos para proseguir con una carrera académica. Por ello se diseñó una investigación que permitiera acercarse de una forma más cualitativa a algunas de estas respuestas.

\section{Metodología}

Con el propósito de tener un primer acercamiento al destino de quienes han participado en el programa y del posible impacto del vic en la formación de investigadores, acotamos el límite temporal de la base de datos del verano, la cual originalmente abarcaba de 1991 a 2012, para quedar de 1991 a 2004. También eliminamos a los participantes de nivel medio superior, para con- 
servar sólo a los participantes de nivel licenciatura. La nueva delimitación dejó libre un periodo de diez años para contrastar la base de datos del Verano 1991-2004 frente al directorio 2014 del sNI, un marco temporal aceptable para que un individuo concluya los estudios de licenciatura, de maestría y de doctorado, bajo la idea de que ya podría estar trabajando como investigador. Como se sabe, el SNI no reúne a la totalidad de investigadores del país, pero es la base de datos más consolidada.

Los resultados arrojaron que la presencia de los exparticipantes en el SNI con respecto a los participantes por año, si bien nunca alcanza 20\%, disminuye conforme pasa el tiempo (Gráfica 3), a pesar del crecimiento en el número de participantes.

Los 477 exparticipantes del VIC, localizados en la base de datos del SNI, reportan una distribución por áreas disciplinarias inversa a la que se presentó en la base de datos del vic: en primer lugar se encuentra el área de ciencias biológicas, biomédicas y químicas con $32 \%$; en segundo, las áreas físico matemáticas con $26 \%$; después las ingenierías y disciplinas relacionadas con la tecnología con $25 \%$; finalmente las ciencias sociales constituyen $17 \%$ del total.

Los programas regionales, a los que se hizo referencia antes, aparecieron a partir de 1996; durante sus cinco primeros años el vic fue el único referente nacional, y justamente observamos una mayor presencia de exparticipantes del Verano en el SNI 2014, por lo que decidimos trabajar con el periodo 19911995 del programa.

Hasta este momento sólo se conocían datos cuantitativos, producto de la comparación de la base de datos del Verano y del SNI, pero se desconocía su trayectoria una vez que concluyeron la licenciatura, cómo se fue construyendo su interés en la investigación, qué eventos ocurrieron en sus trayectorias que les permitieron dedicarse a la investigación, y sobre todo, no se sabía si el hecho de haber realizado la estancia del Verano había tenido algún significado. La logística del programa sólo abarca hasta la conclusión de la estancia, pero hasta el año 2012, del que se tiene información disponible, no se conoce de algún seguimiento formal de exparticipantes. Es por eso que buscamos realizar entrevistas a profundidad, para conocer las subjetividades de aquellos a quienes pudiera localizarse y aceptaran ser consultados.

En un principio localizamos el correo electrónico y enviamos la invitación para entrevistar a exparticipantes miembros del SNI que provinieran de los estados de la república con mayor número de participantes en el programa veraniego, pero debido a que no se obtuvo la respuesta esperada, al final abordamos a quien respondiera la petición y efectuamos un total de 16 entrevistas semiestructuradas, realizadas entre febrero y septiembre de 2016, algunas de manera personal, otras por videoconferencia, o telefónicamente. 


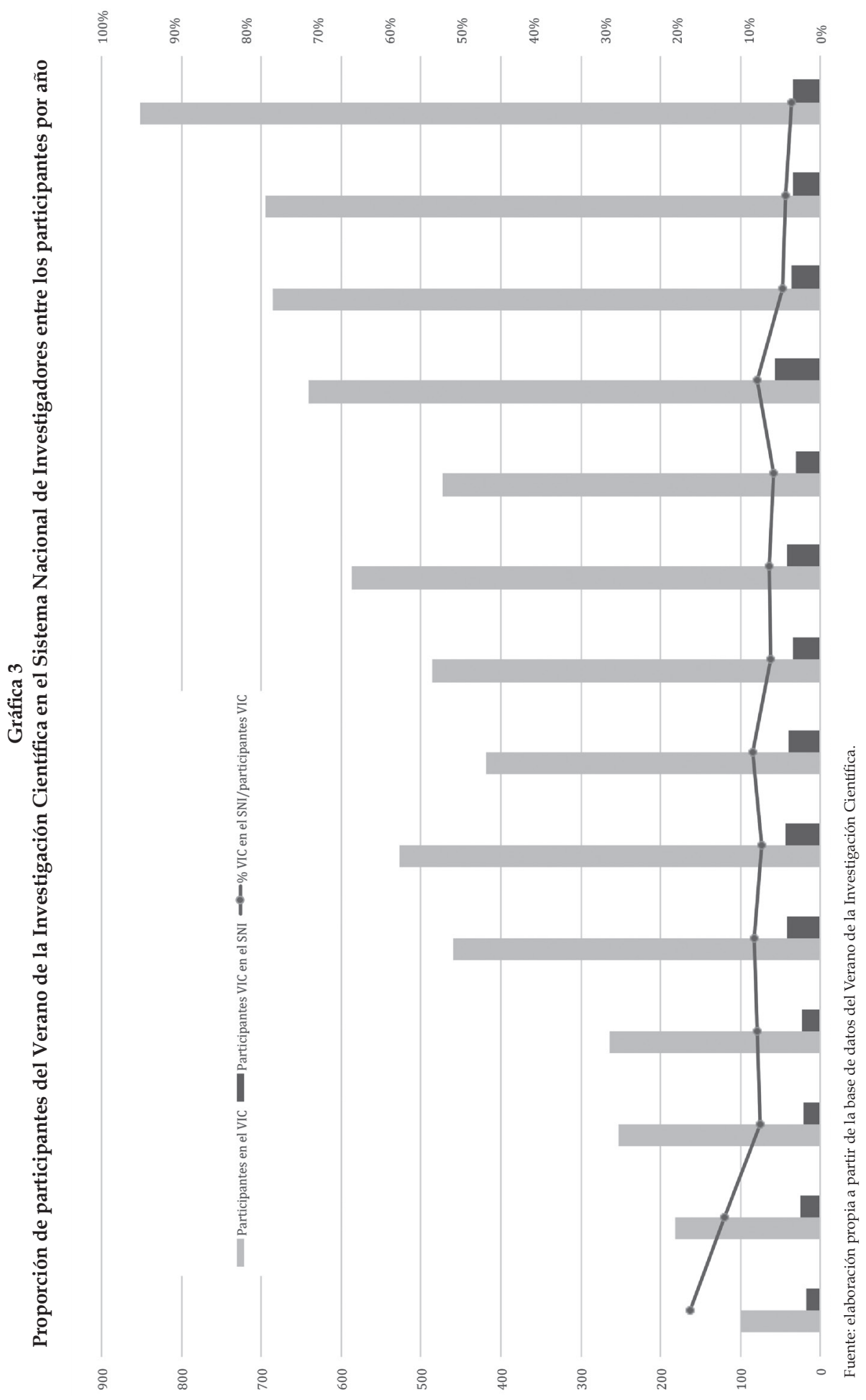


El guión abarcó tres grandes rubros: a) su experiencia sobre sus estudios de licenciatura: entorno familiar, expectativas laborales, situaciones personal y profesional actuales; b) opinión sobre la investigación como profesión y de la formación de recursos humanos para la investigación; y c) sobre la experiencia de su primer contacto con la investigación. Sólo al final de la entrevista les hablábamos del vic y del propósito de la investigación, aclarando todas las dudas que tuvieran. Transcribimos las entrevistas y las codificamos de acuerdo a los rubros arriba mencionados; los testimonios serán reportados de manera anónima para guardar su carácter confidencial.

Tabla 2

Participantes del Verano de la Investigación Científica identificados en el directorio 2014 del Sistema Nacional de Investigadores, que fueron entrevistados

\begin{tabular}{|c|c|c|c|}
\hline Nombre & Carrera & Entidad origen & Entidad de la estancia \\
\hline Adán & Ingenierías & Ciudad de México & Ciudad de México \\
\hline Alberto & Físico matemáticas & Jalisco & Ciudad de México \\
\hline Alejandro & Sociales y humanidades & Jalisco & Colima \\
\hline Ana & Físico matemáticas & San Luis Potosí & Ciudad de México \\
\hline Adriana & Biológicas & Sinaloa & Ciudad de México \\
\hline Alejandra & Biológicas & Morelos & Morelos \\
\hline Adrián & Físico matemáticas & Ciudad de México & Ciudad de México \\
\hline Araceli & Biológicas & Veracruz & Nuevo León \\
\hline Arturo & Biológicas & Jalisco & Estado de México \\
\hline Andrea & Biológicas & Ciudad de México & Yucatán \\
\hline Andrés & Sociales y humanidades & Yucatán & Ciudad de México \\
\hline Angel & Ingenierías & Baja California & Ciudad de México \\
\hline Antonio & Físico matemáticas & Coahuila & Ciudad de México \\
\hline Alma & Físico matemáticas & Sonora & Morelos \\
\hline Alba & Biológicas & Sonora & Chihuahua \\
\hline Abigail & Biológicas & Ciudad de México & Ciudad de México \\
\hline
\end{tabular}

Fuente: elaboración propia a partir de la base de datos del Verano de la Investigación Científica.

\section{Pocos pero... ¡habemus brujos!}

De las respuestas de los entrevistados puede desprenderse que, en general, la estancia influyó de manera determinante para formarse una expectativa laboral relacionada con la actividad de investigación una vez que terminaron la carrera. Casi todos los entrevistados mencionaron al vIC durante la entrevista; al finalizar les comentamos el motivo de nuestra indagatoria y su 
reacción fue positiva con respecto a la experiencia de su estancia, es decir, la labor en la que interactuaron alumno e investigador (aprendiz y maestro) para realizar los trabajos - proceso mediante el cual el estudiante conoció la cotidianeidad de la actividad investigativa - tuvo un significado positivo para ellos.

Identificamos dos condiciones: la primera, y muy frecuente, en la que los entrevistados indicaron que ya tenían nociones de lo que significaban los términos "ciencia" o "investigación", es decir, poseían cierto background o habitus, debido a la interacción con su familia (porque sus mamás los llevaban a museos, o bien en la familia había académicos), con sus profesores (por la forma en que desarrolló su labor docente, por las propias materias o por ser sus auxiliares de investigación), o por el interés personal (por curiosidad o por la lectura de publicaciones de divulgación científica); y la segunda, en la que dos de los entrevistados no habían conocido nada sobre ciencia e investigación sino hasta que participaron en la estancia, lo cual resultó fundamental para que se formaran una expectativa laboral al respecto:

Lo que pasa es que yo participé en el Verano de la Investigación, ahí fue cuando conocí realmente un poquito el ambiente académico... Sí, fue, digamos, como el detonante... De un póster, o sea, no sé, del Verano, cómo está; bueno, antes ponían pósters en las escuelas y ya te inscribías, y ya si te seleccionaban, participabas (Adán, ingenierías, Ciudad de México).

El otro ejemplo es el de una exbecaria (Adriana, biológicas, Sinaloa), quien comentó que en el cuarto año de la carrera conoció la coordinación (academia) de la investigación científica; entonces vino a la Ciudad de México, contactó a algunos académicos y así fue como conoció por primera vez el entorno de la investigación. Señaló que nunca pasó por su cabeza ser investigadora, ya que quería dedicarse a la medicina. El verano marcó su vida.

Aquí resulta interesante destacar el papel del capital cultural de los entrevistados; al momento en que ingresaron a la universidad para estudiar su licenciatura, los padres de la mitad de ellos habían cursado estudios hasta la educación media superior (presentándose todas las posibilidades: sin estudios, primaria inconclusa, secundaria, carrera técnica y bachillerato), mientras que los padres de cinco más contaban con educación normal, licenciatura y posgrado; desconocemos el último grado de estudios de los padres de tres más, aunque los entrevistados señalaron que eran comerciante, ferrocarrilero y trabajador. Una distribución muy parecida se presentó entre sus madres: en el momento en que el entrevistado ingresó a la universidad, casi la mitad de ellas contaba con estudios hasta de nivel medio superior (sin estudios, primaria inconclusa, secundaria, carrera técnica y bachillerato), mientras que 
las madres de seis más contaban con estudios de nivel licenciatura, normal y posgrado; ignoramos el nivel de estudios de las madres de tres de los entrevistados, pero en el caso de dos se reportó que una se dedicaba al hogar y otra al comercio, de la tercera se desconoce su ocupación. Precisamente hablando de ocupaciones, no todos los entrevistados hablaron al respecto, pero encontramos entre los padres a dos maestros normalistas y un profesor universitario, tres en negocio familiar, un ingeniero civil, un contratista, dos empleados de CFE, uno que se dedicaba a la política y un ferrocarrilero; entre las mamás encontramos cinco amas de casa, tres en negocio familiar, dos maestras normalistas, una contadora (ya fallecida) y una profesora de preparatoria. El conjunto de estas circunstancias permite suponer que en la gran mayoría de los casos se trató del primer (y tal vez único) miembro de la familia en dedicarse a la investigación:

[...] digamos, mi padre garantizó hasta la licenciatura [...], curiosamente mi madre estudió hasta la secundaria, pero siempre proveía y buscaba, y se preocupaba porque nosotros tuviéramos los elementos para que siguiéramos estudiando [...]; una de las cosas que garantizó fue que aprendiéramos el idioma inglés [...], entonces a pesar de ser una mujer de esa característica, nunca dejó de estar alrededor de nosotros [...]. En la escuela secundaria, igual, mi madre tuvo que ver porque siempre buscaba las escuelas que tuvieran el mayor ranking en Guadalajara [...]; creo que la figura materna jugó un peso fundamental (Alejandro, sociales y humanidades, Jalisco).

A casi todos los entrevistados, la estancia del vic les permitió conocer tres elementos fundamentales que representan el espíritu del programa: cómo se llevaba a cabo el proceso de la investigación, cómo era un investigador, y visualizar la actividad como una profesión; además, varios aseveraron que identificaron la importancia de la actividad en su proceso de formación metodológica. Una entrevistada menciona que aun cuando ya conocía previamente al académico (astrónomo), el Verano le permitió trabajar de manera más estrecha con él, reconociendo este encuentro como su primer acercamiento a la investigación (Ana, físico matemáticas, San Luis Potosí). Otra exparticipante afirmó lo siguiente:

[...] más bien yo creo que fueron mis profesores quienes me dieron probaditas de esto, con los proyectos, las tareas que me dejaron aquí en la carrera, y en combinación con programas que, yo sí de verdad saqué mucho beneficio de ellos, son los programas estos de Veranos de la Investigación; estos programas en donde en el verano uno tiene la oportunidad de ir un mes a trabajar con un investigador o investigadora y hacer algo más 
apegado a lo que ellos están haciendo como profesionistas; eso me abrió mi panorama de decir 'ah mira, no sabía que uno podía ganarse la vida haciendo eso' (Alma, físico matemáticas, Sonora).

La socialización con otros estudiantes durante la estancia del vic fue un rasgo que enfatizaron de manera importante algunos de los entrevistados, derivada de la propia interacción cotidiana durante la estancia y porque al final de la residencia todos presentaban los resultados de su trabajo en el congreso referido antes, el cual dejó de realizarse para poder proporcionar un mayor número de becas. Así, ellos, en tanto participantes, podían conocer la investigación que se llevaba a cabo en diferentes áreas disciplinarias y les fomentaba un sentido de pertenencia. Nuevamente en referencia a Landesmann (2004), el vínculo aprendiz-maestro había sido relevante y también la relación con otros estudiantes, gente con el mismo interés en la ciencia:

Además, en la licenciatura, había estado en un programa que se llama el Verano de la Investigación Científica, de la Academia Mexicana de Ciencias $[\ldots]$, descubrí cómo era importante ver lo que hacían las otras ciencias, porque a la hora de tener un montón de cuates, pues igual están muy interesados en hacer investigación, pero en Economía, en Biología, en Sociología, lo que sea; me di cuenta de que el entusiasmo que había por buscar cosas nuevas era común a todas las ciencias, que la Física no era muy distinta de eso [...], [ello] me decidió que eso era lo que yo quería hacer (Adrián, físico matemáticas, Ciudad de México).

Otros entrevistados se refirieron a la riqueza del congreso. Adán (ingenierías, Ciudad de México) mencionó que ese espacio le permitió percibir el ambiente académico y que, a partir de eso, decidió cursar una maestría. En palabras de otro entrevistado:

Yo creo que el Verano debería de volver a ser como... bueno, yo creo que fui muy afortunado, fui al tercer verano, porque no nada más era el verano, aparte era la estancia, entonces... era el verano y el congreso donde juntaban a todos los que estuvieron trabajando en el verano de la investigación científica; un congreso que se llamaba la estancia o el congreso del verano, algo así, y a todos nos pusieron a exponer, y todos los que expusimos salimos en este librito, estaba muy chiquitito, ¿no? Pero fue muy bonito, en mi caso, al ver cuánta gente estuvo becada en el área que me tocó a mí, y fueron como cuatro días en donde nos juntaron para que expusiéramos lo que hicimos (Antonio, físico matemáticas, Coahuila).

Y acorde con lo dicho por Grediaga (2012), lo que siguió en el proceso de formación de la mayoría de los exbecarios entrevistados fue la realización 
de estudios de maestría y de doctorado (salvo una excepción en que estudió hasta la maestría), lo cual sucedió, en casi todos los casos, al finalizar la licenciatura. Una segunda etapa de nuestra pesquisa busca indagar sobre qué diferencias existen entre exparticipantes del Verano que no son investigadores y/o miembros del sNI; pretendemos entender mejor la complejidad que involucra dedicarse a la investigación en un país como México. Desde luego que el Verano pudo haber significado un "empujón" hacia la vocación científica, pero de ahí a finalmente convertirse en académico es otra cosa. En las trayectorias de vida suceden múltiples circunstancias que pueden desviar a los sujetos de continuar por esa ruta, muchas veces a pesar de sus propios deseos o planes.

\section{Conclusiones}

Si bien los sujetos que han participado en el programa Verano de la Investigación Científica no conforman una muestra representativa de la matrícula nacional, los datos de sus participantes reflejan en gran medida las cifras nacionales: una mayor participación de las mujeres debido a que se ubican principalmente en carreras asociadas a ciencias sociales y humanidades, y a ciencias biológicas, biomédicas y químicas; en segundo lugar, la participación total por área disciplinaria se ubica en las ciencias sociales y humanidades e ingeniería y tecnología, y por último, el destino de los estudiantes durante su estancia de investigación se concentra en la Ciudad de México. Esta tendencia es decreciente gracias a que cada vez más universidades y centros de investigación participan en el programa para recibir y asesorar a los estudiantes, y a la creación de más instituciones educativas para promover la descentralización de la actividad científica y docente. Esto ha significado que cada año los estudiantes interesados cuentan con una oferta importante de investigadores desarrollando una infinidad de líneas de exploración, lo cual se vuelve un medio de selección respecto a quién resulta más conveniente académicamente para realizar la estancia.

La forma de trabajo bajo la cual opera el programa Verano de la Investigación Científica, basada en la interacción aprendiz-maestro y en la externalización por parte del maestro sobre las habilidades y los valores necesarios para la investigación y la interiorización por parte del aprendiz, puede considerarse artesanal. Parte de un viejo modo tradicional de formación de investigadores ante la falta de una estrategia de reproducción, pero ha representado una importante motivación en quienes fueron entrevistados para este estudio. Los dos aspectos que resaltamos son: el papel que tiene la inte- 
racción con el investigador que los asesoró y la posibilidad de relacionarse con sus pares, incluso cuando la mayoría ya tenía algunas nociones del tema.

No menos importantes resultaron las observaciones en cuanto a la operación del Verano, por ejemplo, con respecto a la suspensión del congreso que se realizaba al final de la estancia, que si bien tuvo como propósito conseguir una mayor cobertura de becas, quitó algo de su espíritu y lo muestra ahora incluso como un programa menos diversificado en comparación con su derivado el programa Delfín. La flexibilidad en el uso de los recursos de cada programa es tema de estudio aparte, pero que hace pensar si el uso de recursos públicos de algún modo limita cualquier iniciativa de innovación. Desde hace más de diez años y hasta la fecha, lo que se lleva a cabo es una reunión de medio curso con los estudiantes que cumplen su estancia en la zona metropolitana del Valle de México (que continúa representando aproximadamente la tercera parte del total de participantes) en la que los estudiantes retroalimentan y, en general, evalúan muy positivamente al programa.

Esta investigación muestra que algunos de los factores para motivar a potenciales investigadores a interesarse por esta actividad van desde las condiciones socioeconómicas, el contexto, las influencias familiares hasta la curiosidad personal y las ganas de aprender, elementos que se entrelazan para ir construyendo este interés por la investigación. Aquí resulta interesante destacar los antecedentes familiares de la mitad de los entrevistados, aquellos padres y madres que aun cuando no contaban con estudios profesionales, sí mostraron un gran interés porque sus hijos continuaran estudiando; sin duda estos padres han tenido una visión más estratégica de la educación que muchos gobernantes en el país. Lo definitivo es que un esfuerzo como el vic puede contribuir de manera relevante a terminar de consolidar ese interés inicial por la investigación y animar a los individuos a dedicarse profesionalmente a ella.

En un país como el nuestro, que se sitúa en los últimos lugares de indicadores internacionales en ciencia y tecnología, el Verano de la Investigación Científica representa un esfuerzo significativo por acercar a los jóvenes hacia la ciencia. Por último, nuestra investigación también expone cómo la formación artesanal de los investigadores sigue siendo uno de los mecanismos más efectivos para transmitir el gusto, el interés y el aprendizaje sobre la ciencia. Después de todo, es el método con el que los brujos aprendían sus trucos, los hechiceros su magia y los profesores medievales enseñaban a sus discípulos. Y mientras no ocurra un cisma en los procesos de enseñanza-aprendizaje, esta manera artesanal de enseñar, al parecer, seguirá siendo la práctica por excelencia de los científicos para formar a sus estudiantes. 


\section{Referencias}

Academia Mexicana de Ciencias (2012). Base de datos 1991-2012 del Verano de la Investigación Científica (acceso restringido).

Asociación Nacional de Universidades e Instituciones de Educación Superior (ANUIES) (2016). Anuario estadístico de educación superior 2014-2015. Fecha de consulta: 6 de octubre de 2016. Recuperado de: http://www.anuies.mx/ informacion-y-servicios/informacion-estadistica-de-educacion-superior / anuario-estadistico-de-educacion-superior

Candela, Antonia (1999). El discurso de la ciencia en el aula. En Remedi, E. (coord.) Encuentros de Investigación Educativa 1995-1998, (pp. 27-53). México: Cinvestav.

Consejo Nacional de Ciencia y Tecnología (Conacyt) (2002). Indicadores de actividades científicas y tecnológicas. México: Edición de bolsillo 2001-2002. Fecha de consulta: 19 de marzo de 2014. Recuperado de : http:/ / www.siicyt.gob.mx/ index.php/estadisticas/informe-general

Consejo Nacional de Ciencia y Tecnología (Conacyt) (2009). Informe general del estado de la ciencia y la tecnología 2008. Fecha de consulta: 19 de marzo de 2014. Recuperado de: http://www.siicyt.gob.mx/index.php/estadisticas/informe-general

Consejo Nacional de Ciencia y Tecnología (Conacyt) (2012). Indicadores de actividades científicas y tecnológicas. México: Edición de bolsillo 2012. Fecha de consulta: 19 de marzo de 2014. Recuperado de: http://www.siicyt.gob.mx/ index.php/estadisticas/informe-general

Consejo Nacional de Ciencia y Tecnología (Conacyt) (2014). Informe general del estado de la ciencia, la tecnología y la innovación 2013. Fecha de consulta: 19 de marzo de 2014. Recuperado de: http://www.siicyt.gob.mx/index.php/estadisticas/informe-general

Consejo Nacional de Ciencia y Tecnología (Conacyt) (2017). Informe general del estado de la ciencia, la tecnología y la innovación 2016. Fecha de consulta: 16 de febrero de 2018. Recuperado de: http:/ / www.siicyt.gob.mx/index.php/estadisticas/informe-general.

Consejo Nacional de Ciencia y Tecnología (Conacyt) (2014). Directorio del Sistema Nacional de Investigadores. Fecha de consulta: 14 de enero de 2014. Recuperado de: http://conacyt.gob.mx/index.php/el-conacyt/sistema-nacional-deinvestigadores

De la Fuente, Nancy (2010). Algunos problemas en la formación de investigadores y en el ejercicio de la investigación. Fecha de consulta: 15 de enero de 2015. Recuperado de: http://www.ur.mx/LinkClick.aspx?fileticket=7lsgyNflx6E\%3D\& tabid $=3872 \& \mathrm{mid}=6440 \&$ language $=$ en-US

García, Susana (1999). Los académicos de la unAm: un viejo problema y dos retos para un nuevo milenio. Sociológica, 14 (41), 61-80. Fecha de consulta: 6 de septiembre de 2016. Recuperado de: http://www.revistasociologica.com. $\mathrm{mx} / \mathrm{pdf} / 4104 . \mathrm{pdf}$ 
González, Julia (2013). Apropiarse de un quehacer: la formación de investigadores del Departamento de Biología Celular del Cinvestav. Tesis de maestría, Centro de Investigación y de Estudios Avanzados del Instituto Politécnico Nacional, México.

Grediaga, Rocío (coord.) (2012). Socialización de la nueva generación de investigadores en México. México: ANUIES.

Guerrero, María Eugenia (2007). Formación para la investigación. Studiositas, 2 (2), 3-4.

Landesmann, Monique (2004). La comunidad académica como espacio de socialización de científicos en la UnAM. El caso de los bioquímicos "herederos" de la facultad de Medicina de la unAm (1957-1974). En Remedi, E. (coordinador). Instituciones educativas. Sujetos, historia e identidades, (pp. 59-92). México: Plaza y Valdez Editores.

Magaña, Deneb Elí (2014). Motivaciones y limitantes en la formación en investigación a través del programa de verano científico: un estudio en una muestra de estudiantes universitarios. Revista Internacional Administración y Finanzas, 7 (6), 103-120. Fecha de consulta: 8 de mayo de 2014. Recuperado de: https:/ / papers.ssrn.com/sol3/papers.cfm?abstract_id=2334467

Maldonado, Alma (2017). La inequidad en la producción y el uso del conocimiento ¿dónde queda América Latina?. En Ágora Latinoamericana Construcción de Espacios Regionales: Inclusión Social Latinoamericana, (pp. 57-69). Tomo II. Bogotá: Universidad de Ciencias Aplicadas y Ambientales, Asociación de Universidades de América Latina y el Caribe para la Integración.

Menin, Ovide (2000). La formación de los investigadores jóvenes. Fundamentos en Humanidades, 1 (1), 90-92. Fecha de consulta: 28 de abril de 2014. Recuperado de: http:/ / www.redalyc.org/pdf/184/18400107.pdf

Montiel, María Araceli (2012). Vínculos, transferencias y deseo de saber. Reconstrucción de trayectorias académicas de prestigio: tres casos de la UNAM. Tesis de maestría, Centro de Investigación y de Estudios Avanzados del Instituto Politécnico Nacional, México.

Moreno, María Guadalupe (coordinadora) (2003). Delimitación del campo temático formación para la investigación. En Ducoing, P. (coordinadora) Sujetos, actores y procesos de formación. Tomo I: Formación para la investigación, Los académicos en México, Actores y organizaciones, (pp. 51-57). México: Grupo Ideograma Editores.

Parry, Sharon (2007). Introduction. Disciplines and doctorates, (pp 3-14). Dordrecht: Springer, Higher Education Dynamics.

Ramírez, Vicenta (1998). La figuración productiva: cultura académica de la Facultad de Química. Tesis de maestría, Centro de Investigación y de Estudios Avanzados del Instituto Politécnico Nacional, México.

Rosas, Rocío (2006). Merton y el sillón 41 en los sistemas sociales: la evaluación académica para el emeritazgo. Tesis de maestría, Universidad Nacional Autónoma de México, México.

Sáenz, José Rafael et al. (2013 noviembre). El talento universitario. Análisis sobre el impacto del VIC en la formación de jóvenes investigadores en la UAMCEHuAt. Memorias del 3er. Coloquio Internacional de Educación y Sociedad, con el tema 
"Escenarios emergentes en educación: para una sociedad en la diversidad", y la $6^{a}$. Jornada Binacional de Educación Intercultural, (pp. 82-93). Fecha de consulta: 15 de mayo de 2014. Recuperado de: http:/ / bibliotecadigital.tamaulipas.gob. $\mathrm{mx}$ /archivos/descargas/4aae8fb533ce1fdca26dae2219aa3e9868d1b0cf.pdf

Secretaría de Educación Pública (SEP) (2015). Principales cifras del sistema educativo nacional 2014-2015. México: SEP. Fecha de consulta: 6 de octubre de 2016. Recuperado de: http://www.planeacion.sep.gob.mx/Doc/estadistica_e_indicadores/principales_cifras/principales_cifras_2015_2016_bolsillo_preliminar. pdf

Stekolschik, Gabriel (2007). La comunicación pública de la ciencia y su rol en el estímulo de la vocación científica. Redes, 12 (25), 165-180. Fecha de consulta: 20 de febrero de 2014. Recuperado de: http:/ / www.redalyc.org/articulo. oa?id=90702506

Urrea, María Luisa (2013). Los veranos de la investigación científica. Una vía para la formación de jóvenes investigadores en la Universidad Autónoma de Sinaloa. Ponencia en XI Congreso Nacional de Investigación Educativa. 4) Educación Superior, Ciencia y Tecnología. Fecha de consulta: 2 de junio de 2014. Recuperado de: http://www.comie.org.mx/congreso/memoriaelectronica/v11/ docs/area_04/0718.pdf

Verano de la Investigación Científica (2015). Directorio 2014 del Sistema Nacional de Investigadores (acceso restringido).

Zelzman, Claudia (2009). El rol de la universidad en la construcción de vocaciones científicas. Revista Mexicana de Orientación Educativa, 6 (17), 9-11. Fecha de consulta: 4 de marzo de 2014. Recuperado de: http://www.remo.ws/revistas/remo-17.pdf 